\title{
RESÚMENES DEL TERCER TALLER DE LA RED DE COOPERACIÓN \\ LATINOAMERICANA SOBRE EL ESTUDIO DEL TIZÓN TARDÍO DE LAS SOLANÁCEAS (MAYO 27, 2018, PERÚ)
}

Revista Latinoamericana de la Papa 22 (1): 76 - 87

ISSN: $1853-4961$

http://www.papaslatinas.org/ojs/index.php/index/oai

Análisis genético de la población actual de Phytophthora infestans en Perú

Genetic analysis of the current population of Phytophthora infestans in Peru

\author{
Soledad Gamboa ${ }^{1}$, Willmer Perez ${ }^{1}$, Myriam Izarra ${ }^{1}$, Almendra Astete ${ }^{2}$, Mirian Yanina \\ Correa $^{2}$ and Hannele Lindqvist-Kreuze ${ }^{1}$ \\ 1 International Potato Center, Lima, Peru. \\ ${ }^{2}$ Consultant, Lima, Peru.
}

Potato late blight is one of the most serious threats to potato production worldwide. Phytopthora infestans causes losses between 50-100\% in native potatoes at 4300 masl, indicating adaptation of this pathogen to different environmental conditions. In 2001 (Perez et al.), population analysis in Peru showed only A1 mating type and four clonal lineages: EC1 and US- 1 in cultivated potatoes and PE-3 and PE-7 in native varieties or wild species; EC-1 was predominant. In 2015 (Izarra, et al.), a new lineage was found in the same frequency as EC-1. To monitor these changes and analyze the genetic structure of the current population of P. infestans, a sampling was carried out during 2016-2017 in Central (Huánuco, Junín, Pasco), North (Cajamarca, Ancash, La Libertad, Piura, Amazonas) and South (Cusco) highlands. Samples from alternative solanaceous hosts, growing near the fields with symptoms caused by Phytophthora were also collected. A total of 247 isolates and 502 samples on FTA cards were analyzed. The pathogen was found in seven alternative hosts. All isolates belonged to A1 mating group, mostly resistant to metalaxyl with simple to complex virulence patterns. In cultivated potatoes, mitochondrial haplotype IIa predominated, being Ia less frequently. Meanwhile, in isolates from other Solanaceous the haplotype $\mathrm{Ib}$ was found in the same frequency as IIa. Using multiplex SSR markers a total of 74 alleles were detected over 12 loci. STRUCTURE analysis grouped the isolates in three clusters. New alleles and high variability within EC-1 genotype was detected. 
Phytophthora urerae sp. nov. infectando a una especie nativa de la familia Urticaceae en

\section{Perú}

Phytophthora urerae sp. nov. infecting a native species of family Urticaceae in Peru

Nicklaus J. Grünwald, ${ }^{1,5}$, Gregory A. Forbes ${ }^{2}$, Willmer Perez-Barrera ${ }^{3}$, Jane E. Stewart $^{4}$, Valerie J. Fieland ${ }^{4}$, and Meredith M. Larsen ${ }^{5}$

${ }^{1}$ Department of Botany and Plant Pathology and Center for Genome Biology and Biocomputing, Oregon State University, Corvallis, OR, USA.

2 Consultant.

${ }^{3}$ International Potato Center, Lima, Peru.

${ }_{5}^{4}$ Department of Botany and Plant Pathology, Oregon State University, Corvallis, OR, USA.

${ }^{5}$ Horticultural Crop Research Unit, USDA-ARS, Corvallis, OR, USA.

A new Phytophthora species causing foliar lesions on a native host known as "Ortiga negra or Mula chalanka" (Urera laciniata Godot ex Wedd) was characterized morphologically and phylogenetically using nuclear and mitochondrial loci and ALFP markers. The colonies were smooth and slightly stellate on Rye B and V8, appeared cottony and petallate to stellate on PDA. Sporangia (23-43 $\mu \mathrm{m} \times 13-19 \mu \mathrm{m})$ were readily formed on agar but neither chlamydospores nor oospores were formed in culture media. After pairing tests, oospores (18 $-37 \mu \mathrm{m}$ ) were formed readily with an A1 tester isolate of P. infestans but not with the A2 tester. All inoculations on $U$. laciniata were successful, producing symptoms identical to those observed in the nature. The ITS genotype of $P$. urerae was identical to that of $P$. andina and $P$. mirabilis. However, analyses of nuclear loci and AFLP markers indicated that $P$. urerae is a new species in clade 1c. Findings of new species in South America may provide novel insights into the origin and evolutionary history of clade 1c Phytophthora species. 


\title{
Genotipificación de poblaciones de Phytophthora en Latino América
}

\section{Genotyping of Phytophthora populations in Latin America.}

\author{
María Florencia Lucca ${ }^{1}$, Silvia Restrepo ${ }^{2}$, Ivette Acuña ${ }^{3}$ and Samantha Zanotta ${ }^{4}$ \\ 1 Potato Research Group, National Agricultural Technology Institute (INTA), Balcarce \\ Argentina. \\ 2 Department of Biological Sciences, Universidad de Los Andes, Bogotá, Colombia. \\ 3 Instituto de Investigaciones Agropecuarias (Institute of Agricultural Research), INIA \\ Chile. \\ 4 Biological Institute of São Paulo, Brasil.
}

Phytophthora infestans, the causal agent of late blight, is one of the most costly pathogens of potato productions worldwide. The center of origin of potato and P. infestans is Latin America, where the pathogen coevolved with a large diversity of Solanaceous species. The first historical tragedy associated with $P$. infestans, the Irish potato famine, occurred 170 years ago and even today, remains the main threat of potato crops. After the launched of Tizón Latino Network in 2014 in Bogotá, Colombia, the National Agricultural Technology Institute (INTA) is carrying out a SSR genotyping service and new available genomic strategies that can be applied to the study Phytophthora populations. Training and outreach activities between partner countries are available. Balcarce Research Station and the Genomic Unit of Biotechnology Institute of INTA have received samples from Argentina, Chile, Brazil, and Colombia from different Solanaceae hosts. We analyzed Phytophthora populations with an internationally agreed panel of 12 microsatellite markers used by the Euroblight Network. Results from SSR markers showed that populations of P. infestans in each country are mostly composed of clonal lineages with distinct genotypes. In Argentina, since 2011 the lineage AG-1 (A2 mating type) was displaced by the clonal lineage EU_2_A1. Recent years, US-1 was displaced by EU_2_A1 in Chile. In Colombia, all P. infestans isolates are belonging to the clonal lineage EC-1. Recently, a new Phytophthora species, $P$. betacei, was described infecting tree tomato crops in Southern Colombia (Nariño State). In Brazil, the preliminary analyzes have shown a predominance of the genotype $2 \mathrm{~A} 1$ in potato and US-1 in tomato. The final analysis is underway and will be presented in the workshop. These data will provide important insights into the genetic population dynamics of $P$. infestans populations and helping to develop better management strategies against late blight disease in Latin America. 


\title{
Mejoramiento Genético para obtención de clones elite de papa con resistencia al tizón
} tardío y tolerancia al calor para enfrentar el cambio climático

\section{Genetic breeding to select resistant potato elite clones to late blight and heat tolerance to} face the climate change

\author{
Manuel Gastelo ${ }^{1}$, Carolina Bastos ${ }^{1}$, Catherine Quispe ${ }^{1}$ y Luis Diaz ${ }^{2}$ \\ ${ }^{1}$ Centro Internacional de la Papa, Lima, Perú. \\ ${ }^{2}$ Consultor.
}

El tizón tardío (Phytophthora infestans. Mont de Bary), es la principal enfermedad del cultivo de papa en el mundo, cuando no es controlada adecuada y oportunamente puede causar la pérdida total del cultivo. En la actualidad el cultivo se está expandiendo a otras áreas donde las condiciones no son ideales para la producción, debido a las altas temperaturas, uno de los principales factores que afectan la producción de tubérculos. También el cambio climático está afectando los patrones climatológicos de las áreas tradicionales para este cultivo, donde la presencia de regímenes anormales de lluvia y el incremento de la presión de plagas y enfermedades, incrementan el riesgo a los agricultores. En anticipación a estos cambios, el Centro Internacional de la Papa ha desarrollado la población LBHT, con resistencia al tizón tardío y tolerancia al calor, adaptada a estas nuevas condiciones. En el primer ciclo de selección y recombinación, se han generado 44 clones elite, con altos niveles de resistencia al tizón tardío, virus, alto rendimiento bajo condiciones de altas temperaturas (superiores a las variedades Yungay y Desiré usadas como controles), precoces y bajo contenido de glicoalcaloides. Estos clones están disponibles para ser usados en los países en desarrollos para obtener nuevas variedades o como parentales en sus planes de mejoramiento. En el Segundo Ciclo, seleccionaremos clones que además de la resistencia al tizón tardío, se adapten tanto a condiciones de temperatura alta y normal y toleren a la sequía, de esta manera los agricultores podrían sembrar estas nuevas variedades bajo estreses abióticos y bióticos originados por el cambio climático y lograr la seguridad alimentaria, preservación del medioambiente y la salud humana. 


\section{El tizón de la papa (Phytophthora infestans) en Bolivia: Retos y oportunidades}

\section{Late blight (Phytophthora infestans) in Bolivia: Challenges and opportunities}

Julio Gabriel $^{1,2}$, jury Magne ${ }^{1}$, Silene Veramendi ${ }^{1}$, Ada Angulo ${ }^{1}$, Felix Rodriguez ${ }^{1}$, Carlos Bejarano $^{1}$, Raúl Esprella ${ }^{1}$ y Manuel Manobanda ${ }^{2}$

${ }^{1}$ Fundación PROINPA, Casilla 4285, Cochabamba, Bolivia.

${ }^{2}$ Universidad Estatal del Sur de Manabi, Jipijapa, Manabí, Ecuador.

El oomycete Phytophthora infestans, agente causal del Tizón de la papa, es la enfermedad más importante en Bolivia, debido a su capacidad destructiva, al infectar hojas, tallos y tubérculos, cuando hay condiciones medioambientales favorables, llegando a producir pérdidas de hasta del $100 \%$ en corto tiempo. Con los objetivos de i) conocer las razas, los tipos de apareamiento y las características moleculares, ii) evaluar algunos cultivares mejorados resistentes en estas zonas, y iii) realizar el combate químico; se colectaron cinco aislamientos de las zonas paperas más importantes del país, donde también se implementaron parcelas experimentales (Colomi y Anzaldo - Cochabamba, Tarabuco y Lampacillos en Chuquisaca, Betanzos en Potosí y Colquencha en La Paz) y campañas fitosanitarias en la zona de Morochata. Los resultados mostraron que las razas de tizón fueron complejas y están presentes los 11 genes de virulencia. El tipo de apareamiento según los análisis moleculares detectó el tipo de apareamiento A1, contrario a lo que se reportó hasta ahora. Este nuevo paradigma será conveniente confirmar para desarrollar nuevas estrategias para el combate de este patógeno. Los cultivares Pinker y Cholita Rosada, fueron resistentes $P$. infestans y tuvieron altos rendimientos. Pafrita y Puka Huaycha (resistentes) tuvieron moderado rendimiento. Asimismo, los cultivares Pafrita y Pinker fueron resistentes a la sequía y Cholita Rosada recupero mejor a este factor. El cultivar Huaycha obtuvo los menores rendimientos en todas las localidades ( $>$ a 15 tha-1). El combate químico, a través de las campañas fitosanitarias fue efectivo y benefició a más de 3.000 familias de pequeños agricultores de las regiones de Morochata, Independencia, Colomi y Capinota en Cochabamba, que lograron cosechas mayores a $10 \mathrm{t}$ ha-1, respecto de $6 \mathrm{t}$ ha- 1 que antes se lograba; además, se redujo a un 50\% el uso de plaguicidas, contribuyendo esto a disminuir los efectos en la salud de los agricultores y el medio ambiente.

Agradecimientos: Proyecto financiado por el Subproyecto "Desarrollo de variedades con tolerancia a factores bióticos restrictivos" del INIAF y ejecutado por Fundación PROINPA, y Swisscontact, a través de su proyecto "Promoviendo Mercados Rurales". 
Nuevos clones de papa para enfrentar el cambio climático en la región Huánuco, Perú

New Potato clone to face the climate change in Huanuco region, Peru

Alejandro Mendoza ${ }^{1}$ y Manuel Gastelo ${ }^{2}$.

${ }^{1}$ Consultor, Huánuco, Perú.

2 Centro Internacional de la Papa, Lima, Perú.

El cambio climático ocasiona el incremento de la temperatura, exceso o falta de lluvias, y mayor presión de enfermedades y/o plagas. Las variedades de papa tienen periodo vegetativo entre 5 a 7 meses y son afectadas por periodos de sequía, que coinciden muchas veces con etapas cruciales de la fenología del cultivo como: emergencia de plantas o llenado de tubérculos, causando pérdidas de 50 a $60 \%$ de la producción. También afecta la calidad de los tubérculos, debido al incremento de la temperatura que ocasiona daños en su morfología (deformaciones y crecimiento secundario), y en el contenido de glicoalcaloides. Durante las campañas agrícolas 2015 - 2017, en la región Huánuco se evaluaron en forma participativa, 20 clones provenientes del programa de mejoramiento del Centro Internacional de la papa (CIP) con resistencia a rancha, tolerancia al calor, precocidad (90-110 días) y calidad para procesamiento. Los clones mostraron buen nivel de resistencia hasta el $20 \%$ de infección, mientras que los testigos estuvieron con 80 a $100 \%$ de infección y rendimiento de 30 a 40 t/ha. destacando los clones CIP 308517. 91 y CIP 308499.314. En cuanto a color de fritura (hojuelas y tiras) y contenido de materia seca destacaron los clones CIP 308486.314 y CIP 308517.91 las cuales pueden ser futuras variedades para el Perú. 
Liberación de la variedad "INIA 325 - PODEROSA", ejemplo de esfuerzo conjunto público -privado en la sierra norte del Perú

New potato variety "INIA 325 - PODEROSA", example of successful public -private partnership

\section{José R. Otiniano ${ }^{1}$, Héctor A. Cabrera ${ }^{2}$, Juan M. Pérez ${ }^{1}$, Henry C. Sánchez ${ }^{1}$ y Manuel Gastelo $^{3}$}

${ }^{1}$ ONG Asociación Pataz.

${ }^{2}$ Instituto Nacional de Investigación Agraria (INIA).

${ }^{3}$ Centro Internacional de la Papa (CIP).

INIA 325 - PODEROSA es una variedad resistente al tizón tardío de la papa y se constituye en un claro ejemplo y producto de la alianza estratégica público - privado, en la cual instituciones con intereses comunes se unen para solucionar un problema. En la sierra norte del Perú, sólo en las regiones de La Libertad, Cajamarca y Ancash se siembran anualmente más de 60,000 ha de papa y su principal limitante es el tizón tardío, que incluso puede causar la pérdida total del cultivo. El año 2011, la Asociación Pataz, el Centro Internacional de la Papa (CIP), Instituto Nacional de Innovación Agraria (INIA), la Municipalidad Distrital de Chugay y productores de la zona, unieron sus esfuerzos y empezaron a realizar experimentos participativos para evaluar 13 clones pertenecientes a la población de mejoramiento B1C5 del CIP. Esta población se caracteriza por su alta resistencia al tizón tardío, altos rendimientos y buena calidad culinaria. En diciembre del 2014, este trabajo coordinado dio su fruto al liberarse la variedad "INIA 325-Poderosa" (CIP 399049.22) para beneplácito de los agricultores de la región norte del Perú. Actualmente, como parte de esta alianza públicoprivado se están realizando otros trabajos de selección participativa para obtener variedades de papa con resistencia al tizón tardío, al calor y a las heladas. 
Validación de un Sistema de alerta temprana con pronóstico a tres días para el manejo del Tizón tardío de la papa en la zona sur de Chile

\section{Validation of an early warning system on 3 day-based forecasts for potato late blight management in Southern Chile}

\section{Ivette Acuña ${ }^{1}$, Rodrigo Bravo ${ }^{1}$, Jorge Gatica ${ }^{1}$ y Juan Quintana ${ }^{2}$}

${ }^{1}$ Instituto de Investigaciones Agropecuarias, INIA Chile, Osorno, Región de Los Lagos, Chile.

${ }^{2}$ Dirección Meteorológica de Chile, DMC-Chile, Santiago, Región Metropolitana, Chile.

El Tizón tardío es la enfermedad más importante del cultivo de papa en Chile, por lo que los agricultores utilizan el control químico en forma preventiva, pero en exceso. Como una forma de apoyar las aplicaciones oportunas y sustentables de fungicidas, INIA Chile desarrolló, validó e implementó en el 2007, un sistema de alerta temprana, basada en el modelo Blitecast (http://tizon.inia.cl). Este sistema utiliza información meteorológica en tiempo real, con datos de la red de estaciones INIA, para predecir el riesgo de la enfermedad. El sistema hoy cuenta con 5000 usuarios registrados. Una encuesta refleja que el $45 \%$ de estos usuarios utiliza el sistema para tomar decisiones de aplicación de fungicidas, pero, aún hay un $21 \%$ que aplica a calendario fijo. Esto último dado por el corto período disponible entre la alerta y la aplicación oportuna. Con el objetivo de mejorar la oportunidad y el tiempo de decisiones para las aplicaciones, se ha validado la incorporación al sistema de alerta el uso de información de pronóstico del tiempo a 24, 48 y $72 \mathrm{hrs}$. Se ha comparado la eficiencia de control de tratamientos basados en alerta con pronóstico, versus calendario fijo, alerta actual y testigo absoluto, durante 3 temporadas. No se detectó diferencias significativas en el daño de tizón tardío entre los tratamientos (RAUDPC: 0.06, 0.05, 0.02, 0.01, 0.05 respectivamente), pero, todos fueron mejor que el testigo absoluto (RAUDPC: 0.41). El sistema de alerta logró reducir en $50 \%$ la cantidad de aplicaciones necesarias, en comparación a un calendario fijo. En temporadas con baja presión de la enfermedad, se logró reducir en un $75 \%$ el uso de fungicidas. La incorporación de datos de pronóstico en el sistema de alerta temprana INIA, como herramienta de apoyo a la toma de decisiones, es una buena alternativa para reducir el riesgo de Tizón tardío.

Agradecimientos: Investigación financiada por la Fundación para Innovación Agraria, FIA Chile. 
Conocimiento de los agricultores sobre el manejo del Tizón tardío de la papa en Los Andes centrales del Perú.

\author{
Farmers' knowledge on potato late blight management in Peruvian Central Andes

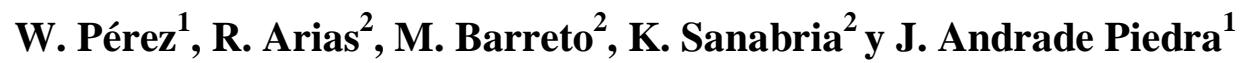 \\ ${ }^{1}$ International Potato Center, Lima Peru. \\ ${ }^{2}$ Consultant.
}

Late blight (LB) has been reported few years ago affecting potato landraces and bred potatoes at higher altitudes in the Andes. This situation, affects mainly to small-scale farmers and puts in risk their food security. However, in places where LB is endemic and potato is cultivated around the year, it is expected that farmers know on disease and its management for the continuous visit of extensionists, agrochemical sellers and staff involved in activities of research and development projects. This study describes farmers' knowledge on LB, its management, and the current practices in two potato growing areas of Peruvian Central highlands. Results of a semi-structured questionnaire to 1198 farmers indicated contradictory results. Despite farmers overwhelmingly considered LB as the main potato disease in both potato growing areas only a few of them mentioned to a fungus as agent causal. Most of them mentioned to rainfall, sunny and foggy days as causing factors of the disease. The use of fungicides based on cymoxanil and mancozeb is the main method to control the disease, however, some of them use insecticides, foliar fertilizers, hormones, and commercial leach as fungicides. Farmers follow indications from pesticide sellers or use their own criteria to apply these products, and do not consider levels of susceptibility of potato varieties or the use of appropriate dosage. Other results are discussed to improve farmers' capacities for better management of LB. 


\section{Desarrollo de un biocontrolador probiótico para el manejo del tizón tardío de la papa} (Phytophthora infestans)

\section{Development of a probiotic biocontrol product for potato late blight management}

$$
\text { Castro, E. }{ }^{1} \text {, Mellado, J.P. }{ }^{1} \text {, Aguayo, M. J. }{ }^{1} \text {, Cerpa, V }{ }^{1} \text { y Moya, E. }{ }^{2}
$$

${ }^{1}$ Laboratorio de Desarrollo de Probióticos, Universidad San Sebastián. Sede Concepción, Chile.

${ }^{2}$ Departamento de Producción Vegetal, Facultad de Agronomía, Universidad de Concepción. Sede Chillán, Chile.

En el ámbito mundial, uno de los cultivos más importante es la papa (Solanum tuberosum), sin embargo, este recurso es afectado por diversas enfermedades, incluyendo el tizón tardío causado por Phytophthora infestans, plaga que puede llegar a ser devastadora, con elevadas pérdidas de las plantaciones. Por esto, una gran diversidad de productos químicos, mayor a los que se aplican en otros cultivos, se emplean en el manejo productivo de este tubérculo. Tendencias más amigables se han incorporado en los últimos años, tales como el uso de control biológico a través de enzimas microbianas que degradan la pared celular de este hongo patógeno, o directamente la aplicación de bacterias biocontroladoras sobre el follaje de la papa. En este contexto, bacterias ácido-lácticas (BAL) se han comenzado a emplear con éxito en el manejo de cultivos agrícolas, donde existe evidencia de los beneficios directos de BAL sobre plantas, las que actúan como agentes nutritivos, tienen efectos antagónicos contra fitopatógenos, promueven la inmunidad y aumentan el crecimiento, rendimiento y calidad de los cultivos. A partir de 120 muestras, se han aislado 98 cepas, las cuales tienen distintos requerimientos de crecimiento, por lo que se cuenta con un pool de Lactobacillus spp. aislados desde suelos, raíces, tallos, hojas, tubérculos y flores de $S$. tuberosum para avanzar en el desarrollo de un biocontrolador para el tizón tardío de la papa.

Agradecimientos: Investigación financiada por CONICYT de Chile. Proyecto FONDEF N $^{\circ}$ ID17AL0035. 


\title{
Sensibilidad a fungicidas sistémicos en aislamientos de Phytophthora betacei y $\boldsymbol{P}$. infestans colectados de tomate de árbol y papa en Colombia
}

Fungicides sensitivity of Phytophthora betacei and $\boldsymbol{P}$. infestans to systemic fungicides in isolates from tree tomato and potato in Colombia

\author{
Mayra Parra', Natalia Guayazán ${ }^{1}$, Giovanna Danies ${ }^{1}$, Maria Fernanda Mideros ${ }^{1}$, \\ Natalia Vargas ${ }^{1}$, Luz Estela Lagos ${ }^{1}$ y Silvia Restrepo ${ }^{1}$. \\ ${ }^{1}$ Universidad de los Andes, Bogotá, Colombia.
}

The genus Phytophthora includes recognized plant pathogen species distributed worldwide which cause devastating diseases that affect many Solanaceae species. Applications of fungicides are often the main method to control these pathogens in the field. Monitoring the development of new resistant populations to common pest molecules is central for designing integrated control strategies. This study focused on assessing the fungicide sensitivity of strains belonging to the species Phytophthora betacei and P. infestans, sampled in localities at the Central and Southern regions of the Andean mountains in Colombia. The main goals were to describe the variation in fungicide sensitivity to cymoxanil, mefenoxam, and fluopicolide by in vitro bioassays within and between two Phytophthora species and collection sites. All strains were molecularly characterized and the genetic population structure was assessed using twelve microsatellite markers. The fungicide in vitro assays showed a diverse response of the strains to the products used. Mefenoxam was the most effective of the three systemic fungicides tested in inhibiting mycelial growth and sporangia of $P$. betacei. Also, a differential control of mycelial growth and sporangia production was observed. The EC50 values tended to be higher when using the sporulation data than when using the mycelial growth data, in addition some isolates showed more mycelial growth and sporulation on media with fungicides. Overall, these findings highlight the importance of monitoring the fungicide resistance level to develop control appropriate management practices in each crop. 


\section{Inductores de resistencia para el manejo de tizón tardío de la papa en Perú}

\section{Resistance inductors for potato late blight management in Peru}

Kimberlayn Sanabria ${ }^{1}$, Willmer Perez ${ }^{2}$, Daniel Sanchez ${ }^{3}$ y Jorge Andrade-Piedra.

${ }^{1}$ Universidad Nacional Agraria La Molina (UNALM).

${ }^{2}$ International Potato Center (CIP), Lima, Perú.

${ }^{3}$ HORTUS S.A., Lima, Perú.

El tizón tardío es el principal factor sanitario limitante del cultivo de papa en todo el mundo y el método más utilizado por los agricultores es el uso de fungicidas. En el presente trabajo determinamos la efectividad y la eficiencia de los inductores de resistencia (IR) para el control del tizón tardío en los andes peruanos. Plántulas de la variedad susceptible Yungay fueron previamente asperjadas con uno de los siguientes IR comerciales: PKplus ${ }^{\circledR}$, Manvert Biolet ${ }^{\circledR}$, Miconic ${ }^{\circledR}$ y Manvert Silikon ${ }^{\circledR}$, para después ser inoculadas con el aislamiento POX067 de Phytophthora infestans bajo condiciones de laboratorio e invernadero. Nuestros resultados reportaron altos valores de protección al follaje con aplicaciones de PKplus ${ }^{\circledR}$ y Manvert Biolet ${ }^{\circ}$. Estos productos fueron seleccionados para ser usados en el experimento de campo en cuatro variedades de papa con diferente grado de susceptibilidad a $P$. infestans en tres zonas durante la época de lluvia. Los tratamientos incluyeron aplicaciones de PKplus®, Manvert Biolet ${ }^{\circledR}$, propineb (solo u alternado); un sistema para la toma de decisiones (DSS, usando fungicidas sistémicos y de contacto) y una parcela sin fungicidas. Las evaluaciones de la severidad de la enfermedad y el área relativa bajo la curva del progreso de la enfermedad (RAUDPC), rendimiento, impacto ambiental (IA) y tasa de retorno marginal (TRM) fueron llevadas a cabo para todos los experimentos. Los más efectivos (bajo RAUDPC, alto rendimiento y bajo IA) y eficientes (TRM>50\%) tratamientos para el control de la enfermedad en las variedades susceptibles fueron obtenidas a través de aplicaciones de PKplus ${ }^{\circledR}$ y aplicaciones de solo propineb, PKplus ${ }^{\circledR}$ alternado con propineb y el uso del DSS. Para la variedad moderadamente resistente, fue con aplicaciones de PKplus ${ }^{\circledR}$ y Manvert Biolet ${ }^{\circledR}$ alternado con propineb, y solo propineb. Para la variedad resistente fue solo con aplicaciones de propineb. Debido a que PKplus ${ }^{\circledR}$ presenta bajos coeficientes de IA, es posible controlar el tizón tardío de la papa de una manera rentable y sostenible con el ambiente, incluso en variedades susceptibles y durante época lluviosa.

Agradecimientos: Proyecto financiado por: R.028-2016-CONCYTEC/SDCTT, Ley 30309. 